\title{
Hyperon-nucleon coupling from QCD sum rules
}

\author{
M.E. Bracco ${ }^{a}$, F.S. Navarra ${ }^{b}$ and M. Nielsen ${ }^{b}$ \\ anstituto de Física, Universidade do Estado do Rio de Janeiro, \\ Rua São Francisco Xavier 524 - 20559-900, Rio de Janeiro, RJ, Brazil \\ bInstituto de Física, Universidade de São Paulo, \\ C.P. 66318, 05389-970 São Paulo, SP, Brazil
}

The $N K Y$ coupling constant for $Y=\Lambda$ and $\Sigma$ is evaluated in a QCD sum rule calculation. We discuss and extend the result of a previous analysis in the $\not i \gamma_{5}$ structure and compare it with the result obtained with the use of the $\gamma_{5} \sigma_{\mu \nu}$ structure. We find a huge violation of the $\mathrm{SU}(3)$ symmetry in the $\gamma_{5} \sigma_{\mu \nu}$ structure.

In understanding the dynamics of kaon-nucleon scattering or the strangeness content of the nucleon using hadronic models, it is important to know the hadronic coupling constants involving the kaons. Among them, $g_{N K \Lambda}$ and $g_{N K \Sigma}$ are the most relevant coupling constants. To determine these couplings using the QCD sum rules 11 one can follow two different approaches: a) the two-point function, where the nucleon and hyperon fields are sandwiched between the vaccum and kaon states, or b) the three-point function where three interpolating fields are sandwiched between vacuum states.

In the case of the pion-nucleon coupling constant, in a pioneer calculation [2 both approaches showed to reproduce the phenomenological value fairly well. However, in this first study the continuum contribution was neglected and since then many calculations were done including higher order terms in the operator product expansion (OPE) and the continuum contribution [3], going beyond the soft-pion limit and including also pole-continuum transitions [4].

For the nucleon-kaon-hyperon coupling constant there are also QCD sum rules calculations based on the two- and three-point functions [5] 7 . The advantages of the three-point function calculation is that it allows for the calculation of the form factors at the hadronic vertices.

In the strange sector, the nucleon-kaonhyperon form factors are used, for instance, to evaluate the strange radius of the nucleon using the kaon cloud [8] and, therefore, a theoretically founded evaluation of these form factors is wellcome.

We will calculate the $g_{N K Y}$ coupling constant using the three-point function

$$
\begin{aligned}
A\left(p, p^{\prime}, q\right) & =\int d^{4} x d^{4} y e^{i p^{\prime} x} e^{-i q y} \\
& \times\left\langle 0\left|T\left\{\eta_{Y}(x) j_{5}(y) \bar{\eta}_{N}(0)\right\}\right| 0\right\rangle
\end{aligned}
$$

where $j_{5}=\bar{s} i \gamma_{5} u$.

As it is well known from two-point sum rules for baryons, there is a continuum of choices for the baryon interpolating fields. Of course the results should be independent of the choice of the current, if we considered an infinity number of terms in the OPE and if we had a perfect model for the continuum contribution in the phenomenological side. However, the OPE has to be truncated and we work with a very simple model for the continuum contribution. Therefore, the results do depend on the choice of the currents. For the proton $\Lambda$ and $\Sigma$ we can write general currents as [9]

$$
\begin{aligned}
& \eta_{P}=2 \varepsilon_{a b c}\left[\left(u_{a}^{T} C d_{b}\right) \gamma_{5} u_{c}+b\left(u_{a}^{T} C \gamma_{5} d_{b}\right) u_{c}\right] \\
& \eta_{Y}=2\left[\eta_{Y_{1}}+b \eta_{Y_{2}}\right]
\end{aligned}
$$

where $b$ is a parameter and

$$
\begin{aligned}
\eta_{\Lambda_{1}} & =\frac{1}{\sqrt{6}} \varepsilon_{a b c}\left[2\left(u_{a}^{T} C d_{b}\right) \gamma_{5} s_{c}+\left(u_{a}^{T} C s_{b}\right) \gamma_{5} d_{c}\right. \\
& \left.-\left(d_{a}^{T} C s_{b}\right) \gamma_{5} u_{c}\right]
\end{aligned}
$$




$$
\begin{aligned}
\eta_{\Lambda_{2}} & =\frac{1}{\sqrt{6}} \varepsilon_{a b c}\left[2\left(u_{a}^{T} C \gamma_{5} d_{b}\right) s_{c}+\left(u_{a}^{T} C \gamma_{5} s_{b}\right) d_{c}\right. \\
& \left.-\left(d_{a}^{T} C \gamma_{5} s_{b}\right) u_{c}\right] \\
\eta_{\Sigma_{1}}= & \frac{1}{\sqrt{2}} \varepsilon_{a b c}\left[\left(u_{a}^{T} C s_{b}\right) \gamma_{5} d_{c}+\left(d_{a}^{T} C s_{b}\right) \gamma_{5} u_{c}\right] \\
\eta_{\Sigma_{2}}= & \frac{1}{\sqrt{2}} \varepsilon_{a b c}\left[\left(u_{a}^{T} C \gamma_{5} s_{b}\right) d_{c}+\left(d_{a}^{T} C \gamma_{5} s_{b}\right) u_{c}\right] .
\end{aligned}
$$

In ref. [9] it was shown that the best interpolating fields for mass sum rules have $b=-1 / 5$. However, to be able to compare our results with previous calculations we will use $b=-1$.

In the phenomenological side the matrix element of the pseudoscalar current between the hyperon and nucleon states defines the pseudoscalar form-factor

$$
\left\langle Y\left(p^{\prime}\right)\left|j_{5}\right| N(p)\right\rangle=g_{P}\left(q^{2}\right) \bar{u}\left(p^{\prime}\right) i \gamma_{5} u(p),
$$

where $u(p)$ is a Dirac spinor and $g_{P}\left(q^{2}\right)$ is related to $g_{N D Y}$ through the relation [2]

$g_{P}\left(q^{2}\right)=\frac{m_{K}^{2} f_{K}}{m_{q}} \frac{g_{N K Y}}{q^{2}-m_{K}^{2}}$,

where $m_{K}$ and $f_{K}$ are the kaon mass and decay constant and $m_{q}$ is the average of the quark masses: $\left(m_{u}+m_{s}\right) / 2$.

Saturating the correlation function Eq.(11) with $Y$ and $N$ intermediate states, and using Eq. (9)we get

$$
\begin{aligned}
& A^{(p h e n)}\left(p, p^{\prime}, q\right)=\lambda_{Y} \lambda_{N} \frac{m_{K}^{2} f_{K}}{m_{q}} \frac{g_{N K Y}}{q^{2}-m_{K}^{2}} \\
& \times \frac{1}{p^{\prime 2}-M_{Y}^{2}} \frac{1}{p^{2}-M_{N}^{2}}\left[\left(M_{Y} M_{N}-p \cdot p^{\prime}\right) i \gamma_{5}\right. \\
& +\frac{M_{Y}+M_{N}}{2} \not i \gamma_{5}-\left(M_{Y}-M_{N}\right) P i \gamma_{5} \\
& \left.-\sigma^{\mu \nu} \gamma_{5} q_{\mu} p_{\nu}^{\prime}\right]+ \text { higher resonances, }
\end{aligned}
$$

where $\lambda_{Y}$ and $\lambda_{N}$ are the couplings of the currents with the respective hadronic states.

In. Eq. (10) we clearly see four distinct Dirac structures. In principle, any of these structures can be used to calculate $g_{N K Y}$ and the sum rules should yield similar results. However, each sum rule may have uncertainties due to the truncation of the OPE side and different contributions from the continuum. Therefore, depending on the Dirac structure we can obtain different results due to these uncertainties. The traditional way to control these uncertainties, and to check the reability of the sum rule, is to choose an appropriate Borel window.

In a recent evaluation of the pion-nucleon coupling constant [10] it was pointed out that the structure $\gamma_{5} \sigma_{\mu \nu} q_{\mu} p_{\nu}^{\prime}$ gives a better result since it gets a smaller contribution from the single pole term coming from $N \rightarrow N^{*}$ transition and it is also independent of the effective model employed in the phenomenological side. Therefore, we will write a sum rule for the $\sigma^{\mu \nu} \gamma_{5} q_{\mu} p_{\nu}^{\prime}$ structure. As we are interested in the value of the coupling constant at $q^{2}=0$, we will make a Borel transform to both $p^{2}={p^{\prime}}^{2} \rightarrow M^{2}$. In Eq. (10) higher resonances refers to pole-continuum transitions as well as pure continuum contribution. The pure continuum contribution will be taken into account as usual through the standard form of ref. 111.

In the OPE side only even dimension operators contribute to the $\sigma^{\mu \nu} \gamma_{5} q_{\mu} p_{\nu}^{\prime}$ structure, since the dimension of Eq.(11) is four and $q_{\mu} p_{\nu}^{\prime}$ take away two dimensions. We will neglect $m_{K}^{2}$ and $m_{s}^{2}$ in the denominators and, consequently, only terms proportional to $1 / q^{2}$ will contribute to the sum rule. We will consider diagrams up to dimension six.

To evaluate the continuum contribution we write a double dispersion relation for the invariant function corresponding to the $\sigma^{\mu \nu} \gamma_{5} q_{\mu} p_{\nu}^{\prime}$ structure:

$$
F\left(P^{2}, P^{\prime 2}, Q^{2}\right)=\int d s d s^{\prime} \frac{\rho\left(s, s^{\prime}, Q^{2}\right)}{\left(s+P^{2}\right)\left(s^{\prime}+P^{\prime 2}\right)} .
$$

The function $\rho\left(s, s^{\prime}, Q^{2}\right)$ (which is proportional to the double discontinuity of $F$ ) can generally be written as

$$
\begin{aligned}
& \rho\left(s, s^{\prime}, Q^{2}\right)=a\left(Q^{2}\right) \delta\left(s-M_{N}^{2}\right) \delta\left(s^{\prime}-M_{Y}^{2}\right)+ \\
& b\left(s^{\prime}, Q^{2}\right) \delta\left(s-M_{N}^{2}\right)+c\left(s, Q^{2}\right) \delta\left(s^{\prime}-M_{Y}^{2}\right)+ \\
& \rho^{\mathrm{OPE}}\left(s, s^{\prime}, Q^{2}\right) \theta\left(s-s_{N}\right) \theta\left(s^{\prime}-s_{Y}\right), \quad(12)
\end{aligned}
$$

where the last term is the tradicional way of taking into account the pure continuum contribution with $s_{N}$ and $s_{Y}$ being the continuum thresholds for the nucleon and hyperon respectively. The 
first term in Eq. (12) gives the double pole contribution and the second and third terms correspond to single pole contributions coming from pole-continuum transitions. In ref. 12] it was shown that single pole contributions are not suppressed by the Borel transformation and give rise to terms proportional to $M^{2}$. Therefore, to take into account the single pole term in the Borel tranformed sum rule we make the substitution:

$g_{N K Y} \rightarrow g_{N K Y}+A M^{2}$,

where $A$ is an unknown parameter. The sum rules we get for $g_{N K \Lambda}$ and $g_{N K \Sigma}$ are [6]

$$
\begin{aligned}
& g_{N K \Lambda}+A M^{2}= \\
& -\sqrt{\frac{2}{3}} \frac{1}{\tilde{\lambda}_{\Lambda} \tilde{\lambda}_{N}} \frac{m_{q}}{m_{K}^{2} f_{K}} \frac{M_{\Lambda}^{2}-M_{N}^{2}}{e^{-M_{N}^{2} / M^{2}}-e^{-M_{\Lambda}^{2} / M^{2}}} \\
& \times a\left[\frac{m_{s} \gamma}{4} M^{2} E_{0}^{\Lambda}-\frac{4}{3} a(1+\gamma)\right], \\
& g_{N K \Sigma}+B M^{2}= \\
& \sqrt{2} \frac{1}{\tilde{\lambda}_{\Sigma} \tilde{\lambda}_{N}} \frac{m_{q}}{m_{K}^{2} f_{K}} \frac{M_{\Sigma}^{2}-M_{N}^{2}}{e^{-M_{N}^{2} / M^{2}}-e^{-M_{\Sigma}^{2} / M^{2}}} \\
& \times \frac{a m_{s} \gamma}{4} M^{2} E_{0}^{\Sigma} .
\end{aligned}
$$

where $a=-(2 \pi)^{2}\langle\bar{q} q\rangle \simeq 0.5 \mathrm{GeV}^{3}, \gamma=$ $\langle\bar{s} s\rangle /\langle\bar{q} q\rangle \simeq 0.8$ and $\tilde{\lambda}_{B}=(2 \pi)^{2} \lambda_{B}$. In the above expressions $E_{0}^{Y}=1-e^{-s_{Y} / M^{2}}$, and this factor in Eqs. (14) and (15) accounts for the continuum contribution. The parameters $A$ and $B$ denotes the contribution from the unknown single pole term coming from $N \rightarrow N^{*}$ transition.

In this calculation the continuum thresholds are chosen to be $s_{Y}=\left(M_{Y}+0.5\right)^{2} \mathrm{GeV}^{2}$. The hadron masses are $M_{N}=0.938 \mathrm{GeV}$, $M_{\Lambda}=1.150 \mathrm{GeV}, M_{\Sigma}=1.189 \mathrm{GeV}$ and $m_{K}=$ $0.495 \mathrm{GeV}$. The strange quark mass is taken to be $m_{s}=150 \mathrm{MeV}$ and the kaon decay constant is $f_{K}=\frac{160}{\sqrt{2}} \mathrm{MeV} \simeq 113 \mathrm{MeV}$. The relevant Borel mass here is $M \simeq \frac{M_{N}+M_{\Lambda}}{2}$ and we analyse the sum rule in the interval $0.8 \leq M^{2} \leq 1.6$ $\mathrm{GeV}^{2}$ where the continuum contribution is always smaller than $50 \%$ of the total OPE.

In Figs. 1 and 2 we show the RHS of Eqs. (14) and (15) as a function of the Borel mass squared (thick solid line). We show the results in a

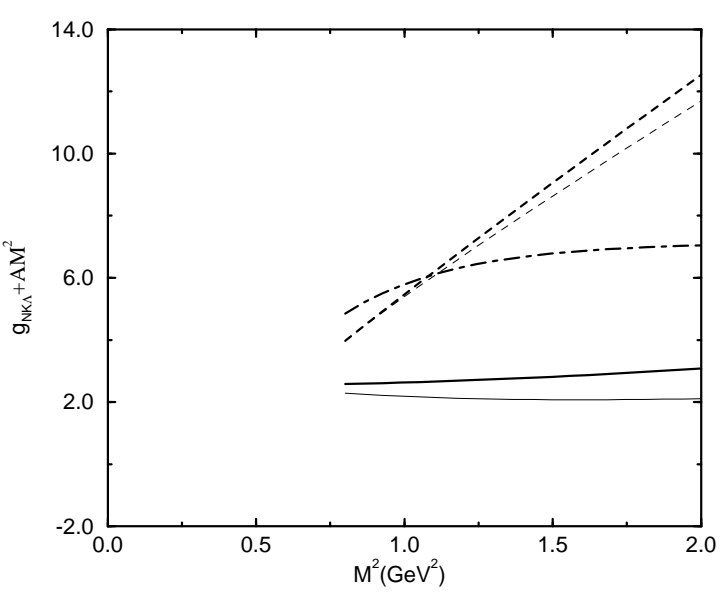

Figure 1. $g_{N K \Lambda}$ as a function of the squared Borel mass $M^{2}$ for the $\gamma_{5} \sigma_{\mu \nu}$ structure (solid line) and for the $\not \gamma_{5}$ structure with (dashed line) and without (dotdashed line) continuum contibutions. The thick lines are obtained using the continuum thresholds given by: $s_{\Lambda}=\left(M_{\Lambda}+0.5\right)^{2} \mathrm{GeV}^{2}$, while for the thin lines lines we used $s_{\Lambda}=\left(M_{\Lambda}+0.7\right)^{2} \mathrm{GeV}^{2}$.

broader Borel range than discussed above to show that our conclusions are not very constrained by the Borel window used. To check the sensitivity of our result on the continuum contribution, we have increased the continuun thresholds as: $s_{Y}=\left(M_{Y}+0.7\right)^{2} \mathrm{GeV}^{2}$ and plotted the corresponding result as the thin line in the same figures. As a first sign it seems that the result is very sensitive to the continuum thresholds. However, as the value of the coupling constant is obtained by the extrapolation of the line to $M^{2}=0$, we imediately see that both curves lead to approximately the same result. Indeed, fitting the QCDSR result to a straight line we get

$$
\left|g_{N K \Lambda}\right|=2.4 \pm 0.1 ;\left|g_{N K \Sigma}\right|=0.03 \pm 0.02,
$$

where the errors were estimated by using the two different thresholds. The values of $A$ and $B$ are very small showing that the single pole contribution is not very important in this structure, in agreement with the results in ref.[10]. The results obtained in $[5]$ are : $\left|g_{N K \Lambda}\right|=6.96$ 


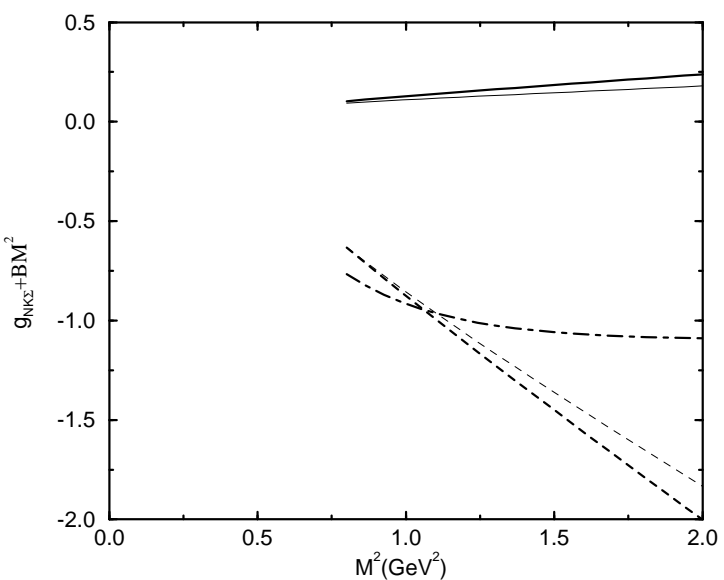

Figure 2. Same as Fig. 1 for $g_{N K \Sigma}$.

and $\left|g_{N K \Sigma}\right|=1.05$. However, the results in ref. 55] were obtained without considering continuum contribution and they are shown as the dotdashed lines in Figs. 1 and 2. Once the continuum contribution is included, through the usual $E_{i}$ factors, the behaviour of the sum rule as a function of the Borel mass changes drastically, as can be seen by the dashed line in Figs. 1 and 2 . In particular, both $g_{N K \Lambda}$ and $g_{N K \Sigma}$ become approximately linear functions of $M^{2}$, showing the importance of the pole-continuum contribution in this structure.

Fitting the RHS of the sum rule results on the structure $\not i \gamma_{5}$ [5] (including the continuum contribution) to a straight line one gets

$$
\left|g_{N K \Lambda}\right|=1.5 \pm 0.3 ;\left|g_{N K \Sigma}\right|=0.25 \pm 0.05
$$

where the errors are again evaluated by considering the two different continuum thresholds.

As in ref. 10 we find out that we can obtain very different results for the coupling constants depending on the structure considered. Of course the procedure used here to extract the coupling constant (fitting the QCDSR result to a straight line in a given Borel window and extrapolating it to $M^{2}=0$ ) is more reliable when the single pole term is small. Therefore, the results obtained for the structure $\not i \gamma_{5}$ may contain big errors since the single pole contribution to this structure is very strong, as can be seen by the dashed lines in Figs. 2 and 3. On the other hand, we may say that the results on the structure $\gamma_{5} \sigma_{\mu \nu}$, analysed here, are not contaminated by the single pole transitions and its extraction with the method used here is more reliable.

As a final remark we note that the values for the coupling constants obtained here in both structures considered, are not in agreement with the exact $\mathrm{SU}(3)$ symmetry. In this limit one gets [6] $\left|g_{N K \Lambda} / g_{N K \Sigma}\right|=3.55$. Therefore, our results show a huge breaking of $\mathrm{SU}(3)$ symmetry. One important question is if this breaking of the $\mathrm{SU}(3)$ symmetry is related with the particular choice of the interpolating fields. Work in this direction is in progress [13].

\section{REFERENCES}

1. M.A. Shifman, A.I. Vainshtein and V.I. Zakharov, Nucl. Phys. B120, 316 (1977).

2. L.J. Reinders, H. Rubinstein and S. Yazaki, Phys. Rep. 127, 1 (1985).

3. H. Shiomi and T. Hatsuda, Nucl. Phys. A594, 294 (1995).

4. M.C. Birse and B. Krippa, Phys. Lett. B373, 9 (1996); Phys. Rev. C54, 3240 (1996).

5. S. Choe, M.K. Cheoun and S.H. Lee, Phys. Rev. C53, 1363 (1996); S. Choe, Phys. Rev. C57, 2061 (1998).

6. M.E. Bracco, F.S. Navarra and M. Nielsen, Phys. Lett. B454, 346 (1999).

7. T.M. Aliev, M. Savc1, hep-ph/9902466.

8. H. Forkel, M. Nielsen, X. Jin and T.D. Cohen, Phys. Rev. C50, 3108 (1994).

9. H.G. Dosch, M. Jamin and S. Narison, Phys. Lett. B220, 251 (1989).

10. H. Kim, S.H. Lee and M. Oka, Phys. Lett. B453, 199 (1999); Phys. Rev. D60, 034007 (1999).

11. B.L. Ioffe, Nucl. Phys. B188, 317 (1981); Nucl. Phys. B191, 591 (1981).

12. B.L. Ioffe and A.V. Smilga, Nucl. Phys. B232, 109 (1984).

13. H.G. Dosch, S. Narison, F.S. Navarra and M. Nielsen, work in progress. 